\title{
Methods in Mammary Gland Biology and Breast Cancer Research: An Update
}

\author{
Michael T. Lewis • Weston W. Porter
}

Received: 10 November 2009 / Accepted: 10 November 2009/Published online: 2 December 2009

(C) Springer Science+Business Media, LLC 2009

In addition to the obvious clinical importance of breast cancer research, the mammary gland has emerged as a powerful model system for the study of basic questions in developmental biology. The increased interest in mammary gland research has brought with it an expansion of the in vitro and in vivo methods for experimental analyses.

In the year 2000, a long-needed and exceptionally useful book entitled "Methods in mammary gland biology and breast cancer research", edited by Margot Ip and Bonnie Asch, was published. This book featured a relatively large number of "laboratory friendly" articles that could be used not only by seasoned mammary gland biologists but also, and more importantly, by those new to the field. In the decade since publication, several new methods have been developed that expand our repertoire of techniques for analysis of mammary gland function and development, as well as for evaluation of the behavior of mammary cancers. These techniques include enhanced methods for preparing primary mammary epithelial cells, alternative methods for 3-dimensional culture, and procedures for in vitro and in vivo viral transduction, to name but a few.

This special issue of the "Journal of Mammary Gland Biology and Neoplasia" highlights a few of these novel approaches, and attempts to serve as a partial update of the "Methods in mammary gland biology and breast cancer research" book by presenting select methods in the same" laboratory friendly" style. We hope you find these techniques useful.

\footnotetext{
M. T. Lewis $(\bowtie) \cdot W$. W. Porter

The Lester and Sue Smith Breast Center and Department

of Molecular and Cellular Biology, Baylor College of Medicine,

One Baylor Plaza, BCM600; Room N1210,

Houston, TX 77030, USA

e-mail: mtlewis@breastcenter.tmc.ed

W. W. Porter

Faculty of Toxicology,

Texas A\&M University,

College Station, TX 77843-4458, USA

e-mail: wporter@cvm.tamu.edu
} 